\title{
OPEN Effects of exercise on cervical muscle strength and cross-sectional area in patients with thoracic hyperkyphosis and chronic cervical pain
}

\author{
Hyunghun Moon ${ }^{1}$, Sung-Ki Lee ${ }^{1 \bowtie}$, Won-Moon Kim² \& Yong-Gon Seo ${ }^{3 凶}$
}

There is a lack of studies comparing the effects of different exercise types in patients with thoracic hyperkyphosis. Twenty-four subjects were divided into three groups: corrective exercise, resistance exercise, and physical therapy. The groups performed their respective interventions, two times per week for three months. Clinical outcomes, including the value of Cobb's angle, cervical muscle strength and endurance, and the cross-sectional area of the cervical deep muscles were measured pre- and post-intervention. There was a significant difference in the changes in the thoracic Cobb's angle between the groups $(P<0.001)$. The corrective exercise group revealed a significantly superior increase in muscle strength and endurance between pre- and post-intervention $(P<0.012)$. There was a significant difference in the cross-sectional area of the cervical deep muscles included longus capitis and multifidus between the groups $(P<0.036$ and 0.007 , respectively). The corrective exercise group showed the most significant increase in cross-sectional area between pre- and post-intervention $(P<0.012)$. A corrective exercise program is a more effective intervention than traditional resistance exercise and physical therapy for improving the thoracic Cobb's angle, cervical muscle strength and endurance, and the cross-sectional area of the deep muscles in patients with thoracic hyperkyphosis. Trial registration: KCT0005292.

The spinal curve facilitates body movement through the interaction of joints including the cervical, thoracic, lumbar, sacral, and hip joints, and enables maintaining upright posture while walking against the center of gravity of the human body ${ }^{1}$. The normal spinal curvature in the sagittal plane comprises a lordotic curve formed in the cervical and lumbar regions, and a kyphotic curve in the thoracic and sacral regions. Excessive curvature is defined as an angle of kyphosis $\geq 40^{\circ}$ of the thoracic spine in the sagittal plane, commonly referred to as thoracic hyperkyphosis ${ }^{2}$.

Thoracic hyperkyphosis can cause a compensatory pattern in body movements, contributing to malalignment of the normal spinal curvature, and resulting in musculoskeletal problems such as back pain and dysfunction ${ }^{3}$. An increase in the kyphotic curve is related to malalignment of the cervical curve, such as hyperextension of the upper cervical region and hyperflexion of the lower cervical region, and continuous overload on the cervical vertebrae can cause forward head posture ${ }^{4}$. Forward head posture is associated with the shortening of the cervical extensors and weakness of the cervical flexors, leading to cervical pain due to continuous tension in the peri-cervical region ${ }^{1}$.

Exercise therapy is commonly recommended as an intervention to improve hyperkyphotic curve and physical function in thoracic hyperkyphosis ${ }^{5,6}$. Bautmans et al. ${ }^{7}$ reported that manual therapy for rehabilitation is an effective intervention to improve thoracic function in patients with thoracic kyphosis. Exercise types in previous studies include back extensor strengthening, abdominal strengthening, and postural education, among which the back extensor strengthening exercise is the most common intervention ${ }^{5}$. Progressive back strengthening exercise

\footnotetext{
${ }^{1}$ Department of Sports Medicine, Cha University, 120, Haeryong-ro, Pocheon-si, Gyeonggi-do 11160, Republic of Korea. ${ }^{2}$ Department of Sports Science, Dongguk University, 123, Dongdae-ro, Gyeongju-si, Gyeongsangbuk-do, Republic of Korea. ${ }^{3}$ Division of Sports Medicine, Department of Orthopedic Surgery, Samsung Medical Center, Sungkyunkwan University School of Medicine, 81 Irwon-ro, Gangnam-gu, Seoul 135-710, Republic of Korea. ${ }^{\circledR}$ email: sklee@cha.ac.kr; yongon79@naver.com
} 


\begin{tabular}{|l|l|l|l|l|}
\hline \multirow{2}{*}{ Characters } & \multicolumn{2}{|l|}{ Two active interventions } & One passive & \multirow{2}{*}{} \\
\cline { 2 - 5 } & CEG & REG & CG & \multicolumn{1}{|l}{} \\
\hline Numbers & 8 & 8 & 8 & - \\
\hline Sex (female/male) & $8 / 0$ & $8 / 0$ & $8 / 0$ & - \\
\hline Age $($ years $)$ & $53.38 \pm 5.61$ & $50.00 \pm 4.00$ & $54.25 \pm 6.16$ & .259 \\
\hline Height $(\mathrm{cm})$ & $158.85 \pm 4.29$ & $159.64 \pm 4.19$ & $159.16 \pm 5.32$ & .996 \\
\hline Weight $(\mathrm{kg})$ & $57.40 \pm 5.56$ & $57.41 \pm 3.66$ & $56.68 \pm 4.44$ & .941 \\
\hline Body mass index $\left(\mathrm{kg} / \mathrm{m}^{2}\right)$ & $22.73 \pm 1.69$ & $22.59 \pm 2.06$ & $22.44 \pm 2.34$ & .910 \\
\hline Neck pain $($ score $)$ & $7.50 \pm 0.54$ & $7.63 \pm 1.06$ & $7.25 \pm 0.46$ & .588 \\
\hline
\end{tabular}

Table 1. Characteristics of study subjects. Data are means \pm standard deviation. CEG, corrective exercise group; REG, resistance exercise group; CG, control group. $P$ values are from Kruskal-Wallis test.

contributes to a significant decrease in thoracic kyphosis and increase in muscle strength and endurance ${ }^{8}$. Katzman et al. ${ }^{6}$ reported that thera-band exercise for enhancing strengthening is beneficial intervention to reduce angle of thoracic hyperkyphosis.

Various exercise intervention have been reported for thoracic hyperkyphosis, and corrective exercise also was reported as an intervention method attributed to improve spinal posture, balance, and well-being in older women with thoracic hyperkyphosis ${ }^{9}$. Corrective exercise included resistance training, stretching, and posture education is associated with reduction of kyphosis angle and thoracic posture ${ }^{10,11}$. According to previous studies, the different types of exercise prescriptions are associated with different clinical outcomes for improving hyperkyphotic curve, muscle strength, and quality of life $e^{10,12,13}$. However, most of studies reported have focused on reduction of thoracic kyphosis and improvement of muscle strength and the studies of comparison of effectiveness between different exercise interventions have not reported.

Therefore, this study aimed to investigate the effects of different exercise types on the thoracic Cobb's angle, and cervical muscle strength and endurance, and to confirm an increase in the cross-sectional area (CSA) of the cervical muscles. Finally, this study aimed to determine the most optimal exercise type for enhancing cervical muscle strength and endurance in patients with thoracic hyperkyphosis with chronic cervical pain.

\section{Results}

The characteristics of the subjects in this study did not show significant differences in baseline data (Table 1).

In result of Cobb's angles, there was a significant difference in the changes in the Cobb's angle of the thoracic curve between the groups (Kruskal-Wallis test, $P<0.000$ ). According to the results of post hoc test, CEG showed a significantly higher than REC and CG (Mann-Whitney U test; CEG vs. REG, $P<0.007$; CEG vs. CG, $P<0.001$ ). REG was showed more improvement than CG $(P<0.003)$. The two groups with active interventions, CEG and REG, showed a significant improvement (Wilcoxon singed rank test, $P<0.012, P<0.011$, respectively), while CG did not show a significant difference between these periods $(P<0.317)$.

In result of the maximal cervical muscle strength, there was a significant difference in the changes between the groups (Kruskal-Wallis test, $P<0.038$ ). The two active intervention groups, CEG and REG, had a significant increase between pre- and post- intervention (Wilcoxon signed-rank test, $P<0.012$ and $P<0.012$, respectively) but not revealed a significant difference in CG $(P<0.231)$. The results of Mann-Whitney $\mathrm{U}$ test for post hoc test, there was no significant difference between the group (CEG vs. REG, $P<0.14$; CEG vs. CG, $P<0.031$; REG vs. CG, $P<0.073)$.

In the muscle endurance of $80 \%$ and $50 \%$, the results revealed that there was a significant difference in the change between the groups (Kruskal-Wallis test, $P<0.000$ ). In the results of post hoc test, CEG showed a higher improvement than REG and CG (Mann-Whitney $U$ test, $P<0.004$ and $P<0.001$, respectively) and REG was more improved than CG $(P<0.015)$ in muscle endurance of $80 \%$. In result of Wilcoxon singed-rank test, CEG and REG has showed a significant improvement after intervention $(P<0.011$ and $P<0.012$, respectively) but not improved in CG $(P<0.914)$ (The change of 50\% muscle endurance in CEG showed also higher than REG and GC (Mann-Whitney U test, $P<0.001$ and $P<0.001$, respectively) but not showed a significant difference between REG and CG $(P<0.035)$. In results of Wilcoxon signed-rank test, CEG and REG has showed a significant improvement after intervention $(P<0.011$ and $P<0.021$, respectively) but not improved in $\mathrm{CG}(P<0.667)$ (Fig. 1).

In change of CSA, Longus colli was not showed a significant different between three groups $(P<0.424)$. The change after intervention revealed in CEG (Wilcoxon signed-rank test, $P<0.012$ ) but not showed in REG and CG $(P<0.134$ and $P<0.401$, respectively). There was a significant difference between three groups in change of longus capitis (Kruskal-Wallis test, $P<0.036$ ) but not revealed a significant difference between CEG and CG (Mann-Whitney U test, $P<0.046)$, and REG and CG $(P<0.916)$, except CEG and REG $(P<0.016)$. In analysis of Wilcoxon signed-rank test, CEG and REG was showed a significant improvement after intervention $(P<0.012$ and $P<0.012$, respectively) but not showed a significant improvement in CG $(P<0.779)$. In change of multifidus CSA, there was a significant difference between the groups (Kruskal-Wallis test, $P<0.007$ ). In post hoc test, the two active intervention, CEG and REG, has showed a significant different compared with CG (Mann-Whitney U test ; CEG vs. CG, $P<0.016$ and REG vs. CG, $P<0.009$, respectively) but not showed between CEG and REG $(P<0.074)$. The change after intervention revealed in CEG and REG (Wilcoxon signed-rank test, $P<0.012$ and 

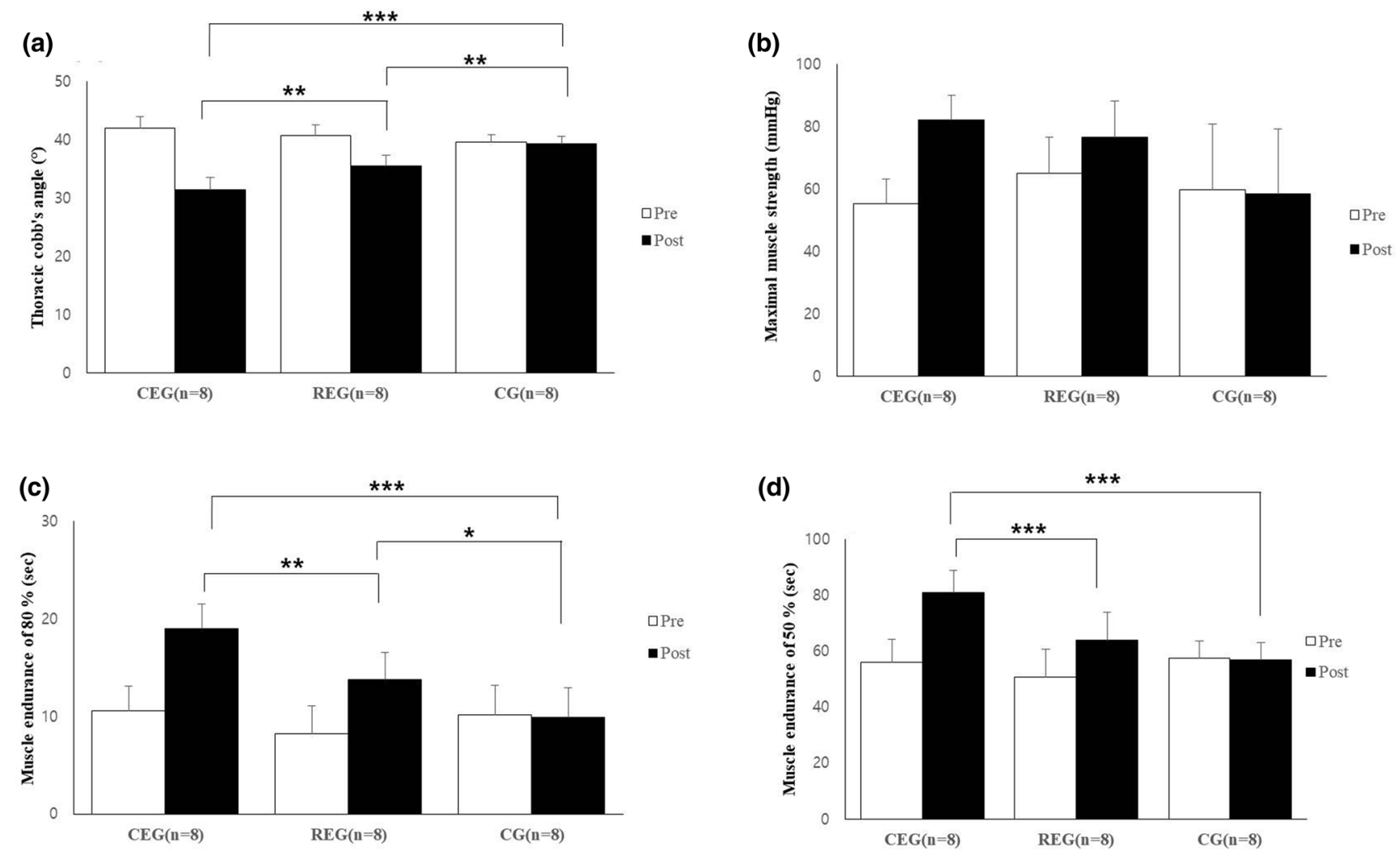

Figure 1. The difference of thoracic angle and cervical muscles strength and endurance after intervention. (a) Thoracic Cobb's angle; (b) Maximal muscle strength; (c) Muscle endurance of $80 \%$; (d) Muscle endurance of 50\%. CEG, corrective exercise group; REG, resistance exercise group; CG, control group. $P<0.0167^{\star}$, $P<0.00167^{\star *}$ : the results from Bonferroni correction.

$P<0.012)$ but not showed in CG $(P<0.123)$ (Fig. 2). Table 2 shows the results after exercise intervention between the groups.

\section{Discussion}

This study compared the effects of different exercise types on Cobb's angle of the thoracic curve, as well as on the strength and endurance of cervical muscles, and the CSA of the cervical deep muscles in patients with thoracic hyperkyphosis. The corrective exercise program (CEP) was more effective in improving Cobb's angle, cervical muscle strength and endurance, and CSA than resistance exercise program (REP) or CG..

Excessive thoracic hyperkyphosis is associated with misalignment of the spinal curve, resulting in forward head posture and cervical pain ${ }^{1,4}$. This study revealed that the corrective exercise and resistance training were beneficial interventions to improve thoracic hyperkyphosis angle. This result is supported by a study by Katzman et al. ${ }^{6}$ which reported that multidimensional group exercise for 12 weeks improved muscle strength and decreased thoracic Cobb's angle by $6^{\circ}$. Additionally, previous systematic reviews ${ }^{5}$ also demonstrated that muscle strength exercise could improve thoracic Cobb's angle in hyperkyphosis of the thoracic vertebrae. Particularly, there was a significant improvement in the thoracic kyphosis angle in CEG when compared to REG. The corrective exercise applied in this study was an integrated exercise program and included mobilization exercise and Schroth method, while resistance exercise only included weight training. Mobilization exercise and the Schroth method can reduce the angle of thoracic hyperkyphosis ${ }^{7,13-15}$. Foad et al. ${ }^{11}$ reported that corrective exercise is a more effective intervention than a general exercise program for patients with thoracic hyperkyphosis which supports the present findings. Therefore, corrective exercise, including mobilization and the Schroth method, is considered as an exercise intervention to improve thoracic Cobb's angle in hyperkyphosis. These results explain why an integrated CEP is more effective than REP included only resistance training. However, further studies including patients with osteoporosis and vertebral fractures have to confirm if these results are applicable for the patients with osteoporosis, vertebral fractures, and kyphosis.

The thoracic spinal column functions as a supporting base for the cervical spine and influences cervical kinematics through the cervicothoracic junction ${ }^{1}$. Concomitant thoracic spinal motion is necessary to produce the complete range of movements at the cervical spine, which are often reduced with changes in the normal alignment of the thoracic spine ${ }^{16}$. An excessive thoracic kyphotic curve is associated with shortness of the cervical extensors and weakness of the cervical flexors ${ }^{4}$. In this study, CEG and REG showed improvement of cervical muscle strength and endurance after exercise therapy for 3 months, and these results are consistent with a study conducted by Kjellman et al. ${ }^{17}$ which reported that resistance training in patients with neck pain could improve static muscle strength and muscle endurance of the cervical muscles. Hakkinen et al. ${ }^{18}$ also reported 
(a)

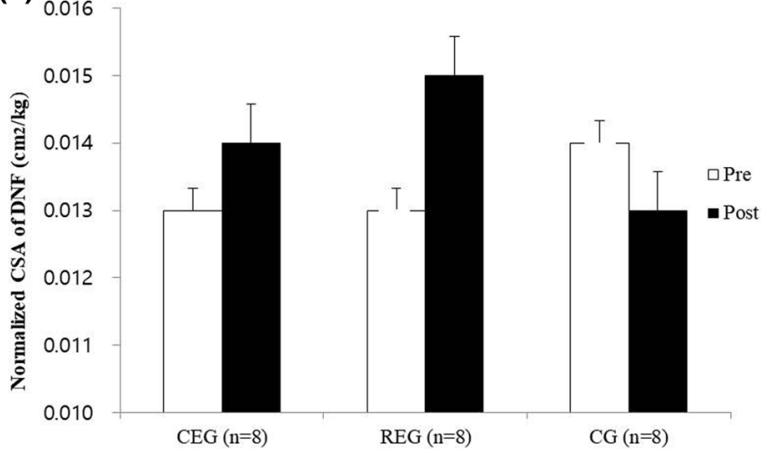

(b)

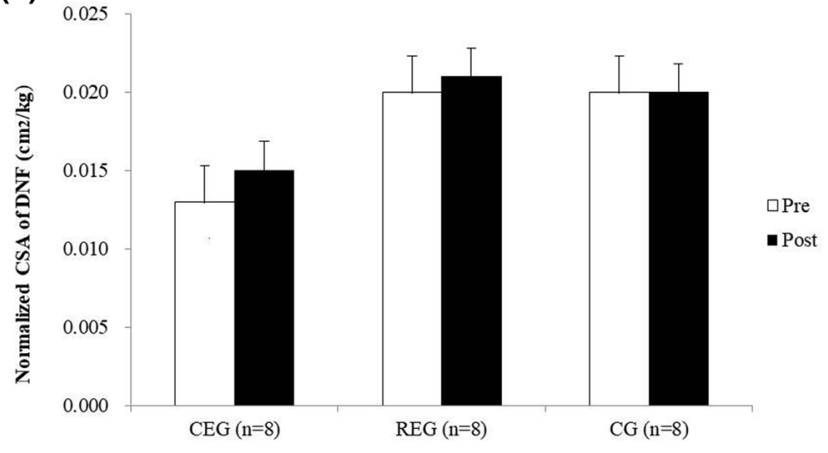

(c)

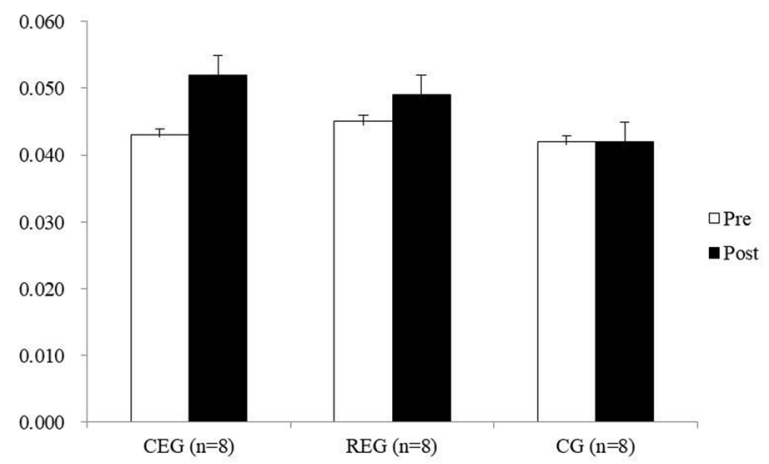

Figure 2. The difference of cross-sectional area in cervical deep muscles after intervention. (a) Longus colli muscle; (b) Longus capitis muscle; (c) Multifidus muscle. CEG, corrective exercise group; REG, resistance exercise group; CG, control group; CSA, cross-sectional area; DNF, deep neck flexor. $P<0.0167^{\star}, P<0.00167^{\star *}$ : the results from Bonferroni correction.

\begin{tabular}{|c|c|c|c|c|c|}
\hline \multirow[b]{2}{*}{ Variable } & \multirow[b]{2}{*}{ Time } & \multicolumn{2}{|c|}{ Two active interventions } & \multirow{2}{*}{\begin{tabular}{|l|} 
One passive \\
CG
\end{tabular}} & \multirow[b]{2}{*}{$P$} \\
\hline & & CEG & REG & & \\
\hline \multirow{2}{*}{ Thoracic Cobb's angle $\left(^{\circ}\right)$} & Pre & $42.00 \pm 2.00$ & $40.75 \pm 1.83$ & $39.63 \pm 1.30$ & \multirow{2}{*}{$.000^{* * *}$} \\
\hline & Post & $31.50 \pm 2.78^{\dagger}$ & $35.50 \pm 2.29^{\dagger}$ & $39.25 \pm 1.04$ & \\
\hline \multirow{2}{*}{ Maximal muscle strength $(\mathrm{mmHg})$} & Pre & $55.3 \pm 88.02$ & $65.13 \pm 11.61$ & $59.88 \pm 21.04$ & \multirow{2}{*}{$.038^{*}$} \\
\hline & Post & $82.13 \pm 12.42^{\dagger}$ & $76.63 \pm 10.59^{\dagger}$ & $58.38 \pm 23.10$ & \\
\hline \multirow{2}{*}{ Muscle endurance of $80 \%$ (sec) } & Pre & $10.63 \pm 2.50$ & $8.25 \pm 2.82$ & $10.13 \pm 3.09$ & \multirow{2}{*}{$.000^{* * *}$} \\
\hline & Post & $19.00 \pm 2.73^{\dagger}$ & $13.75 \pm 2.82^{\dagger}$ & $9.88 \pm 2.17$ & \\
\hline \multirow{2}{*}{ Muscle endurance of $50 \%(\mathrm{sec})$} & Pre & $56.00 \pm 8.04$ & $50.63 \pm 10.06$ & $57.50 \pm 6.23$ & \multirow{2}{*}{$.000^{* * *}$} \\
\hline & Post & $80.88 \pm 9.42^{\dagger}$ & $63.88 \pm 5.84^{\dagger}$ & $56.75 \pm 7.48$ & \\
\hline \multirow{2}{*}{ Longus colli $\left(\mathrm{cm}^{2}\right)$} & Pre & $0.74 \pm 0.12$ & $0.83 \pm 0.15$ & $0.77 \pm 0.16$ & \multirow{2}{*}{.424} \\
\hline & Post & $0.81 \pm 0.13^{\dagger}$ & $0.86 \pm 0.15$ & $0.76 \pm 0.15$ & \\
\hline \multirow{2}{*}{ Longus capitis $\left(\mathrm{cm}^{2}\right)$} & Pre & $0.75 \pm 0.23$ & $1.15 \pm 0.19$ & $1.14 \pm 0.32$ & \multirow{2}{*}{$.036^{*}$} \\
\hline & Post & $0.83 \pm 0.23^{\dagger}$ & $1.18 \pm 0.21^{\dagger}$ & $1.14 \pm 0.32$ & \\
\hline \multirow{2}{*}{ Multifidus $\left(\mathrm{cm}^{2}\right)$} & Pre & $2.48 \pm 0.34$ & $2.57 \pm 0.23$ & $2.37 \pm 0.31$ & \multirow{2}{*}{$.007^{\star *}$} \\
\hline & Post & $2.97 \pm 0.46^{\dagger}$ & $2.76 \pm 0.19^{\dagger}$ & $2.36 \pm 0.30$ & \\
\hline
\end{tabular}

Table 2. The clinical outcomes after exercise intervention of 3 months between the groups. Mean \pm standard deviation. CEG, corrective exercise group; REG, resistance exercise group; CG, control group. ${ }^{\dagger} P<0.05$, ${ }^{\dagger \dagger} P<0.01,{ }^{\dagger \dagger} P<0.001$ : the results from Wilcoxon singed rank test. ${ }^{\star} P<0.05,{ }^{\star \star} P<0.01,{ }^{* *} P<0.001$ : the results from Kruskal-Wallis test.

similar results, showing an increase in cervical strength and endurance by applying resistance training. Both interventions included the same types of exercise, including back muscle strengthening exercises; however, CEP is an integrated program that includes stretching and mobilization. Several studies demonstrated that CEP is a beneficial intervention to improve Cobb's angle and posture in thoracic hyperkyphosis ${ }^{10,11,19,20}$. According to 
those studies, the integrated exercise program could be considered as a specific exercise intervention to obtain more effective results with cervical muscle strength and endurance in patients with thoracic hyperkyphosis.

The cervical deep muscles, including the longus colli, longus capitis, and multifidus, have an important role in the control and stability of the cervical vertebrae ${ }^{21}$. Thoracic hyperkyphosis is associated with misalignment of the cervical curve and this could cause dysfunction of the cervical deep muscles, resulting in chronic cervical pain ${ }^{4}$. Patients with chronic neck pain were shown to have more severe deep muscle atrophy than healthy subjects $^{6}$. Therefore, this study measured the CSA of deep muscles of the cervical vertebrae to compare the effects of different exercise types on CSA. According to the results of this study, the CSA of cervical deep muscles showed a significant improvement in the two active intervention groups. These results are similar to those of a study conducted by Cagnie et al. ${ }^{22}$ which demonstrated that cervical flexion exercises can increase the CSA of cervical deep muscles such as the longus colli and longus capitis. An improvement in CSA may be associated with improvement in muscle strength and endurance of the cervical deep muscles by applying cervical flexor strength exercises. Longus colli was only showed a significant improvement after intervention in CEG and it can be explained the CEP is superior intervention than REG and CG. The CSA of Longus capitis and multifidus were increased in CEG and REG but CEG was showed more improvement than REG. The explanation for this is that the decreased thoracic kyphosis contributes to reduce the overload at the cervical vertebrae and to increase cervical muscle activity, resulting in improvement of CSA in the cervical deep muscles. This mechanism can be explained on the basis of previous studies reporting the positive effects of Schroth and mobilization exercise on muscle activity ${ }^{14,15}$. We suggest that combined corrective exercise should be considered as a more efficient exercise type for increasing CSA of cervical muscles, compared with traditional resistance exercise.

This study has some limitations. Firstly, the subjects were all female. This aspect should be considered because the prevalence of hyperkyphosis in women or men could influence the results. Therefore, this result is not generalizable to all populations, and further study is needed to confirm the difference between male and female subjects. Secondly, the study had a small sample size although it was divided into three different groups. Thus, it is difficult to apply these results to all patients with thoracic hyperkyphosis. A large sample study is needed to confirm these results. Third, this study was not measured the strength of back muscles despite of back strengthening exercise was applied in two exercise groups.

In conclusion, corrective and resistance exercises are beneficial interventions for improving thoracic Cobb's angle, cervical muscle strength and muscle endurance, and the CSA of the cervical deep muscles. These exercises yield better results compared with simple physical therapy. This study revealed that a CEP is a more effective intervention than traditional resistance exercise for improving these parameters. Therefore, these exercise types should be considered when exercise intervention is prescribed to patients with thoracic hyperkyphosis.

\section{Methods}

Study design. This study was a randomized controlled trial with single blind in the participants. It included 38 female patients with thoracic hyperkyphosis with chronic cervical pain diagnosed by an orthopedist. The diagnostic criteria were as follows: chronic neck pain on cervical posterior and upper trapezius muscles for 6 months, kyphosis angle $\geq 38^{\circ}$ of Cobb's angle, and a score $>7$ on the visual analog scale concerning cervical pain. Of these patients, 14 were excluded due to no exercise recommendation from the doctor since three subjects had disc herniation and three were diagnosed with osteoporosis and eight patient refused participation in this study. Twenty-four patients were recruited and informed consent was obtained. Inclusion criteria were: participants having no surgical history within the last three months, no orthopedic and neurological problems, and no experience with exercise therapy for thoracic kyphosis or neck pain. The participants were randomly allocated according to their order of registration in the study to three groups, namely two active intervention groups [the CEG $(n=8)$ and REG $(n=8)$ ], and one passive group [CG $(n=8)$ ]. The study protocol was approved by the Sunmoon University Institutional Review Board (IRB No. SM-201603-004-2) and complied with the 1964 Helsinki declaration and its later amendments.

Outcome assessments. A baseline assessment was conducted before randomization and re-assessment was performed after 12 weeks using the same method. The primary outcomes were Cobb's angle, muscle strength, and endurance of the cervical muscle. The secondary outcome was the CSA of the cervical deep muscles including the longus colli, longus capitis, and multifidus muscles.

Cobb's angle. The change in Cobb's angle was measured by a professional radiologist at a local medical hospital. Participants were instructed to stand with a straight chest and back, with both arms crossed on the chest, and X-rays were taken in the sagittal view. Cobb's angle was measured using Cobb's method, which involves drawing a parallel line at the upper thoracic endplate of the second thoracic vertebrae and lower thoracic endplate of the twelfth thoracic vertebrae, and then erecting perpendiculars from these lines to cross each other ${ }^{23}$ (Fig. 3).

Muscle strength and endurance. Changes in muscle strength and endurance were assessed using a Stabilizer Pressure Biofeedback unit (Stabilizer pressure, Chattanooga, USA). The subjects lay comfortably with knee flexion of $90^{\circ}$, and the device was placed and on the upper cervical spine. Participants were instructed their jaw to push down onto the neck and to hold the resistance in the craniocervical flexion direction against the handheld dynamometer. The reference pressure was set at $40 \mathrm{mmHg}$, which was the average pressure at which the subject could contract the deep neck flexors without discomfort. The maximum cervical strength and endurance were measured as the time to maintain $80 \%$ and $50 \%$ of maximal muscle strength ${ }^{24}$. Trials of the maximum cervical muscle strength were conducted 3 times with 30-s rest time between each trial. After resting 


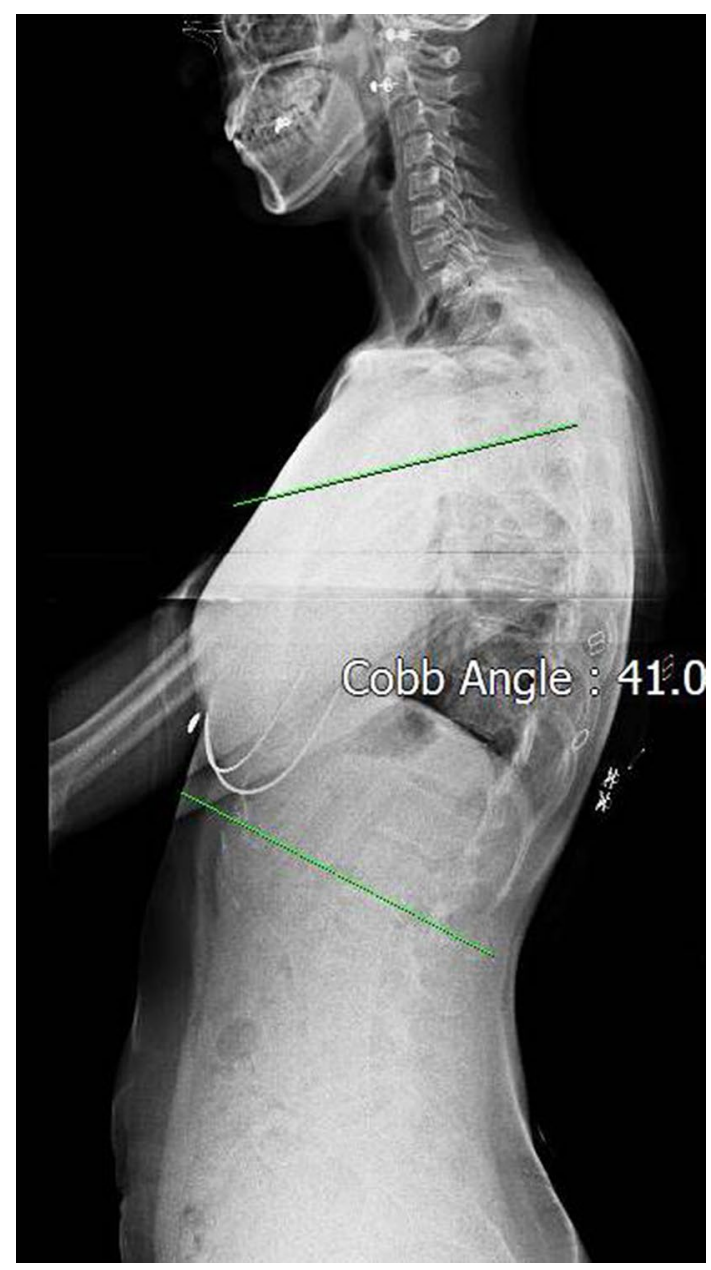

Figure 3. The measurement of Cobb's angle using picture achieving and communication system (PACS).

for $1 \mathrm{~min}$, the subject was instructed to maintain $80 \%$ of the maximum strength within $84.24 \mathrm{mmHg}$ for CEG, $92.10 \mathrm{mmHg}$ for REG, and $87.90 \mathrm{mmHg}$ for CG. This was measured three times. The subject tried the same method at $50 \%$ of maximum strength within $67.65 \mathrm{mmHg}$ for CEG, $72.57 \mathrm{mmHg}$ for REG, and $69.94 \mathrm{mmHg}$ for CG after resting for $1 \mathrm{~min}$. The subject did not complain of pain during the test. The measurement was stopped when the error of the pressure gauge was within $\pm 2 \mathrm{mmHg}$, and recorded that time. The average value of the three measurements was used for the data analysis.

Cross-sectional area of the cervical deep muscles. The CSA of the cervical deep muscles was measured using 1.5 T magnetic resonance imaging (Intra, Philips, Netherlands) before and after intervention. To measure the CSA of the cervical muscles, a T2 image was obtained and used by cutting the transverse axis between the upper and lower endplate of the 5 th to 6 th cervical vertebrae at $5 \mathrm{~mm}^{2}$ thickness and $10 \mathrm{~mm}^{2}$ interval. The image was analyzed using a picture archiving and communication system by an experienced radiologist of 10 years to enhance intra-reliability of the measurement. The CSA of the muscles were calculated by setting and drawing the regions of interest to the right and left of the cervical vertebrae muscles. The right side was used for the analysis (Fig. 4).

Interventions. In this study, correction and resistance exercises were applied to the two active intervention groups, and the passive group was CG. The total exercise time was $60 \mathrm{~min}$ including $5 \mathrm{~min}$ of warm-up, $50 \mathrm{~min}$ of the main exercise, and $5 \mathrm{~min}$ of cool down. Each session was conducted 3 times a week for 12 weeks. The CEP was a modified program from previous studies ${ }^{11,14}$. The CEP is an integrated intervention that includes stretching, mobilization, and Schroth method, which is different with REP. However, the CEP and REP consisted of the same back extensor training. This program focused on a decrease in the thoracic kyphosis angle, improvement of lung function and enhancement of respiratory muscle strength through chest or abdominal breathing training, and improvement of neck pain and function. The exercise intensity was set using a rating perceived exertion scale, which ranged from 13 (somewhat hard) to 15 (hard). The resting time was $30 \mathrm{~s}$ between each set and $60 \mathrm{~s}$ between exercise items. The REP was applied by modifying several resistance programs reported ${ }^{13,16}$. The exercise intensity was measured by evaluating one repetition maximum (1 RM) for each exercise, and the intensity 


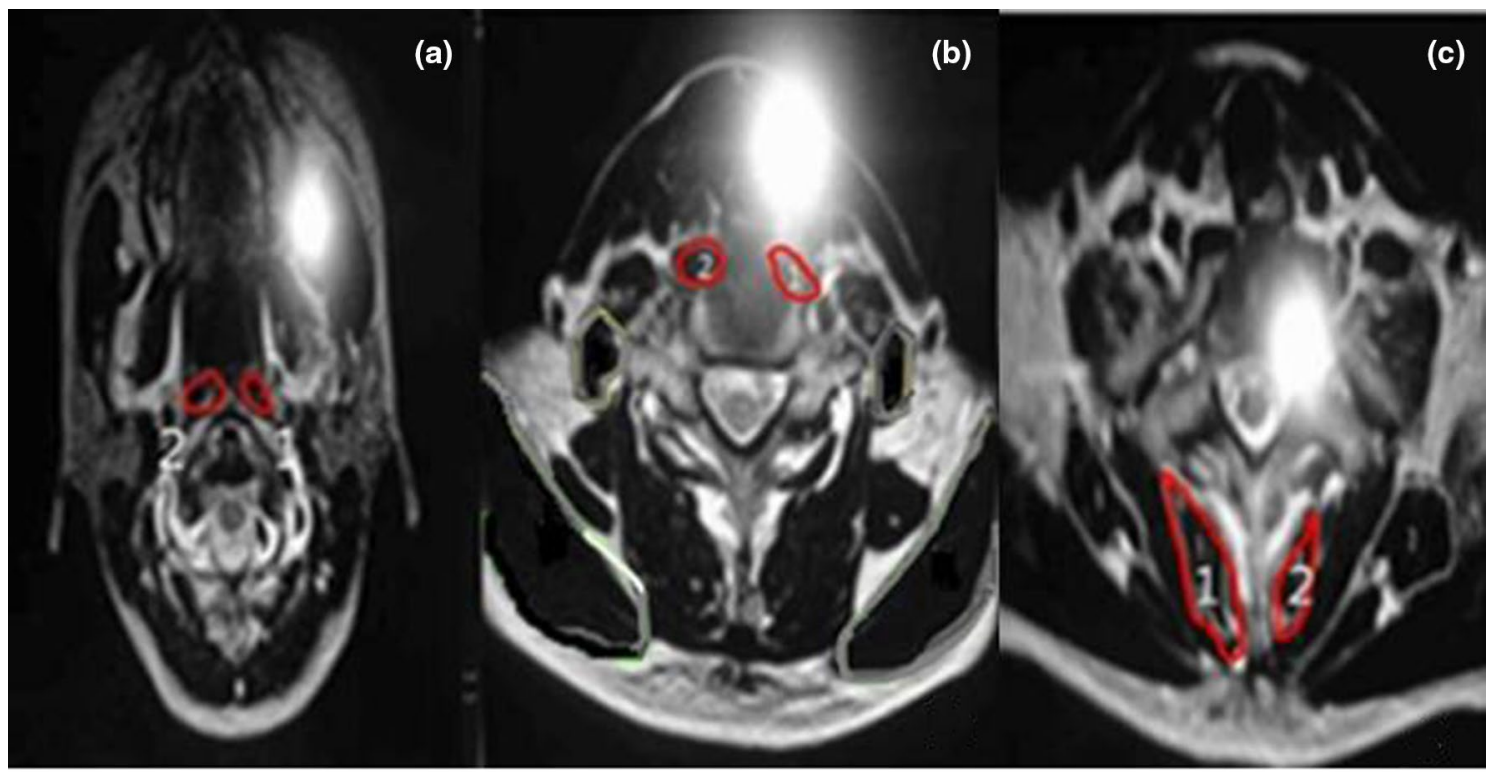

Figure 4. MRI (magnetic resonance imaging) imaging of the cross-sectional area of the perivertebral space. (a) Longus colli muscle; (b) Longus capitis muscle; (c) Multifidus muscle.

\begin{tabular}{|c|c|c|c|}
\hline Exercise types & Exercise modes & Time & Intensity \\
\hline \multicolumn{4}{|l|}{ Corrective exercise program } \\
\hline Cervical corrective exercise & $\begin{array}{l}\text { Cervical retraction exercise }(4 \mathrm{~min}) \\
\text { Cervical mobilization exercise }(4 \mathrm{~min}) \\
\text { Cervical flexion exercise }(4 \mathrm{~min})\end{array}$ & $12 \mathrm{~min}$ & $\begin{array}{l}\text { 20R / 3Sets } \\
\text { RPE 13-15 }\end{array}$ \\
\hline Thoracic corrective exercise & $\begin{array}{l}\text { Thoracic extension exercise }(4 \mathrm{~min}) \\
\text { Thoracic mobilization exercise }(4 \mathrm{~min}) \\
\text { Scapula adduction exercise }(3 \mathrm{~min}) \\
\text { Shoulder horizontal abduction exercise }(3 \mathrm{~min})\end{array}$ & $14 \mathrm{~min}$ & $\begin{array}{l}\text { 20R / 3Sets } \\
\text { RPE 13-15 }\end{array}$ \\
\hline Back extensor exercise & $\begin{array}{l}\text { Trunk extension exercise }(3 \mathrm{~min}) \\
\text { Arm and leg raise exercise }(3 \mathrm{~min})\end{array}$ & $6 \mathrm{~min}$ & $\begin{array}{l}\text { 20R / 3Sets } \\
\text { RPE 13-15 }\end{array}$ \\
\hline Latissimus dorsi and pectoralis stretching & $\begin{array}{l}\text { Foam roller stretching }(3 \mathrm{~min}) \\
\text { Gymball stretching }(3 \mathrm{~min})\end{array}$ & $6 \mathrm{~min}$ & $\begin{array}{l}\text { 20R / 3Sets } \\
\text { RPE 13-15 }\end{array}$ \\
\hline Schroth method exercise & $\begin{array}{l}\text { Kneeling between chair }(4 \mathrm{~min}) \\
\text { Standing in a doorway }(4 \mathrm{~min}) \\
\text { Sitting question mark }(4 \mathrm{~min})\end{array}$ & $12 \mathrm{~min}$ & $\begin{array}{l}\text { 20R / 3Sets } \\
\text { RPE 13-15 }\end{array}$ \\
\hline Warm-up and cool down & Stretching for lower and upper body & $10 \mathrm{~min}$ & RPE 13 \\
\hline \multicolumn{4}{|l|}{ Resistance exercise program } \\
\hline Cervical resistance exercise & $\begin{array}{l}\text { Cervical retraction with thera-band }(4 \mathrm{~min}) \\
\text { Cervical bridge exercise }(3 \mathrm{~min}) \\
\text { Cervical lift off exercise }(3 \mathrm{~min})\end{array}$ & $15 \mathrm{~min}$ & $1 \mathrm{RM}$ of $30 \%$ \\
\hline $\begin{array}{l}\text { Shoulder and scapular muscles strengthen exercise } \\
\text { with thera-band }\end{array}$ & $\begin{array}{l}\text { Scapular retraction }(4 \mathrm{~min}) \\
\text { Lat pull-down }(4 \mathrm{~min}) \\
\text { Seated low rowing }(3 \mathrm{~min}) \\
\text { Prone lying with arm elevator }(4 \mathrm{~min})\end{array}$ & $15 \mathrm{~min}$ & $1 \mathrm{RM}$ of $30 \%$ \\
\hline Posture exercise & $\begin{array}{l}\text { Back extension with weight backpack ( } 5 \mathrm{~min}) \\
\text { Four point kneeling }(5 \mathrm{~min})\end{array}$ & $10 \mathrm{~min}$ & $1 \mathrm{RM}$ of $30 \%$ \\
\hline Leg strength exercise & $\begin{array}{l}\text { Half squat }(5 \mathrm{~min}) \\
\text { Step ups }(5 \mathrm{~min})\end{array}$ & $10 \mathrm{~min}$ & $1 \mathrm{RM}$ of $30 \%$ \\
\hline Warm-up and cool down & Stretching for lower and upper body & $10 \mathrm{~min}$ & RPE 13 \\
\hline
\end{tabular}

Table 3. The exercise program was divided into two active intervention groups including corrective and resistance exercise. $R M$ repetition maximum, $R P E$ rating of perceived exertion.

was set at $30 \%$ of $1 \mathrm{RM}$. The resting time was applied in the same way as that of the CEP. The intervention for CEG was applied CEP while REG was conducted REP (Table 3).

The CG with one passive group was treated by the therapy consisted of thermal stimulation, using an electric heat pack, and ultrasound therapy. The thermal therapy was applied using an electric heat pack for 25 min by setting the temperature at $50^{\circ}$ to $55^{\circ}$, and ultrasound therapy used an Inter current therapy (ITO, Japan) for 
$15 \mathrm{~min}$ at $100 \mathrm{~Hz}$, and a frequency of $1 \mathrm{MHz}$ and intensity of $1.5 \mathrm{~W} / \mathrm{cm}^{2}$ for $10 \mathrm{~min}$, with a total treatment period of $50 \mathrm{~min}$.

Statistical methods. The Kolomgrov-Smirnov test was used to check normal distribution of the data. The baseline characteristics of the three groups were compared using Kruskal-Wallis test, and the mean and standard deviation were used for the descriptive statistics of all the variables in the groups. Kruskal-Wallis test was used to confirm the different effects of each exercise type. The Mann-Whitney U test was used when a significant difference was confirmed and Bonferroni correction was applied with the alpha level set at $P=0.0167$. The Wilcoxon signed-rank test was used to compare all the variables between the pre-intervention and the post-intervention. All the data were analyzed using Statistical Package for Social Sciences (SPSS) 18.0 statistics program for Windows. $P$ values $<0.05$ were considered statistically significant.

\section{Data availability}

The datasets generated during and/or analyzed during the current study are not publicly available due to the institutional ethical regulations of Sunmoon University but are available from the corresponding author on reasonable request.

Received: 20 August 2020; Accepted: 1 February 2021

Published online: 15 February 2021

\section{References}

1. Lau, K. T., Cheung, K. Y., Chan, M. H., Lo, K. Y. \& Chiu, T. T. Relationships between sagittal postures of thoracic and cervical spine, presence of neck pain, neck pain severity and disability. Man Ther. 15, 457-462 (2010).

2. Macagno, A. E. \& O’Brien, M. F. Thoracic and thoracolumbar kyphosis in adults. Spine. 31, S161-S170 (2006).

3. Nault, M. L. et al. Relations between standing stability and body posture parameters in adolescent idiopathic scoliosis. Spine 27, 1911-1917 (2002).

4. Quek, J., Pua, Y. H., Clark, R. A. \& Bryant, A. L. Effects of thoracic kyphosis and forward head posture on cervical range of motion in older adults. Man Ther. 18, 65-71 (2013).

5. Bansal, S., Katzman, W. B. \& Giangregorio, L. M. Exercise for improving age-related hyperkyphotic posture: a systematic review. Arch. Phys. Med. Rehabil. 95, 129-140 (2014).

6. Katzman, W. B., Sellmeyer, D. E., Stewart, A. L., Wanek, L. \& Hamel, K. A. Changes in flexed posture, musculoskeletal impairments, and physical performance after group exercise in community-dwelling older women. Arch. Phys. Med. Rehabil. 88, 192-199 (2007).

7. Bautmans, L., Van Arken, J., Van Mackelenberg, M. \& Mets, T. Rehabilitation using manual mobilization for thoracic kyphosis in elderly postmenopausal patients with osteoporosis. J. Rehabil. Med. 42, 129-135 (2010).

8. Itoi, E. \& Sinaki, M. Effect of back-strengthening exercise on posture in healthy women 49 to 65 years of age. Mayo Clin. Proc. 69, 1054-1059 (1994).

9. Solberg, P. A. et al. Effects of different types of exercise on muscle mass, strength, function and well-being in elderly. Eur. J. Sport Sci. 13, 112-125 (2013).

10. Kamli, F., Shirazi, S. A., Ebrahimi, S., Mirshamsi, M. \& Ghanbari, A. Comparison of manual therapy and exercise therapy for postural hyperkyphosis: a randomized clinical trial. Physiother. Theory Pract. 32, 92-97 (2016).

11. Foad, S., Reza, R., Ismail, E., Mohamad, H. A. \& Hooman, M. The efficiency of corrective exercise interventions on thoracic hyperkyphosis angle. J. Back Musculoskelet Rehabil. 27, 7-16 (2014).

12. Bennell, K. L. et al. Effects of an exercise and manual therapy program on physical impairments, function and quality-of-life in people with osteoporotic vertebral fracture: a randomized, single-blind controlled pilot trial. BMC Musculoskelet. Disord. 11, 36 (2010).

13. Jang, H. J., Hughes, L. C., Oh, D. W. \& Kim, S. Y. Effects of corrective exercise for thoracic hyperkyphosis on posture, balance, and well-being in older women: a double-blind, group-matched design. J. Geriatr. Phys. Ther. 42, E17-E27 (2019).

14. Lehnert-Schroth, C. Three-Dimensional Treatment for Scoliosis. The Schroth Orthopedic Breathing System. A Physiotherapeutic Method to Improve Deformities of the Spine 1st edn. (Ciando, Munich, 2007).

15. Park, S. Y. \& Shim, J. H. Effect of 8 weeks of Schroth exercise (three-dimensional convergence exercise) on pulmonary function, Cobb's angle, and erector spinae muscle activity in idiopathic scoliosis. J. Korea Converg. Soc. 5, 61-68 (2014).

16. Bergström, I., Bergström, K., Karlsson, S., Grahn, K. A. \& Brinck, J. Back extensor training increases muscle strength in postmenopausal women with osteoporosis, kyphosis and vertebral fractures. Adv. Physiother. 13, 110-117 (2011).

17. Kjellman, G. \& Oberg, B. A randomized clinical trial comparing general exercise, Mckenzie treatment and a control group in patients with neck pain. J. Rehabil. Med. 34, 183-190 (2002).

18. Hakkinen, A., Salo, P., Tarvainen, U., Wiren, K. \& Ylinen, J. Effect of manual therapy and stretching on neck muscle strength and mobility in chronic neck pain. J. Rehabil. Med. 39, 575-579 (2007).

19. Feng, Q., Wang, M., Zhang, Y. \& Zhou, Y. The effect of a corrective functional exercise program on postural thoracic kyphosis in teenagers: a randomized controlled trial. Clin. Rehabil. 32, 48-56 (2018).

20. Oxland, T. R. Fundamental biomechanics of the spine-what we have learned in the past 25 years and future directions. J. Biomech. 49, 817-832 (2016).

21. Boyd-Clark, L. C., Briggs, C. A. \& Galea, M. P. Muscle spindle distribution, morphology and density in longus colli and multifidus muscle of the cervical spine. Spine 27, 694-701 (2002).

22. Cagnie, B., Hodge, R. D., Achten, E., Cambier, D. \& Danneels, L. A magnetic resonance imaging investigation into the function of the deep cervical flexors during the performance of craniocervical flexion. J. Manipul. Phys. Ther. 33, 286-291 (2010).

23. Horng, M. H., Kuok, C. P., Fu, M. J., Lin, C. J. \& Sun, Y. N. Cobb angle measurement of spine from X-ray images using convolutional neural network. Comput. Math. Methods Med. 2019, 1-18 (2019).

24. Jull, G. A., Falla, D. B., Treleaven, J., Hodges, P. \& Vicenzino, B. Retraining cervical joint position sense: the effect of two exercise regimes. J. Orthop. Res. 25, 404-412 (2007).

\section{Author contributions}

H.H.M., Y.G.S., and S.K.L. designed the study, and H.H.M performed the study and recorded the data. H.H.M. and Y.G.S analyzed the data and prepared the table and figures. H.H.M. and Y.G.S. contributed to the preparation of the manuscript. S.K.L., W.M.K., and Y.G.S. interpreted the results. All authors critically revised the manuscript and approved the final manuscript. 


\section{Competing interests}

The authors declare no competing interests.

\section{Additional information}

Correspondence and requests for materials should be addressed to S.-K.L. or Y.-G.S.

Reprints and permissions information is available at www.nature.com/reprints.

Publisher's note Springer Nature remains neutral with regard to jurisdictional claims in published maps and institutional affiliations.

(c) (i) Open Access This article is licensed under a Creative Commons Attribution 4.0 International License, which permits use, sharing, adaptation, distribution and reproduction in any medium or format, as long as you give appropriate credit to the original author(s) and the source, provide a link to the Creative Commons licence, and indicate if changes were made. The images or other third party material in this article are included in the article's Creative Commons licence, unless indicated otherwise in a credit line to the material. If material is not included in the article's Creative Commons licence and your intended use is not permitted by statutory regulation or exceeds the permitted use, you will need to obtain permission directly from the copyright holder. To view a copy of this licence, visit http://creativecommons.org/licenses/by/4.0/.

(c) The Author(s) 2021 